\title{
On the Geometry of Our Universe
}

\author{
Anantha Anantha
}

C/O Siddhartha Kalingarayar, Zamin Uthukkuli P.O, Pollachi, Tamil Nadu 642004, India

Received: 1 October 2018, Accepted: 29 October 2018

Published online: 19 February 2019.

\begin{abstract}
According to Freedman's Equations, The Geometry of the Universe may be Euclidean, Lobachevskian or Riemannian. The recent probes of Boomerang experiments, WMAP and Planck revealed that our universe is nearly flat. But till this day, there is no mathematical formulation for the geometry of our Universe. In this article, by applying the laws of Classical Algebra, the author attempts to show that the shape of our Universe is purely flat.
\end{abstract}

Keywords: Fate of The Universe, Cosmic Triangles, Freedman Equations

\section{Introduction and results}

\section{Case 1}

Given: Cosmic Triangle ABC.

Construction: Make $A D=A E ; A F=A G$ and $A H=A J$. Join $\mathrm{D}$ and $\mathrm{E} ; \mathrm{F}$ and $\mathrm{G}$ and $\mathrm{H}$ and $\mathrm{J}$

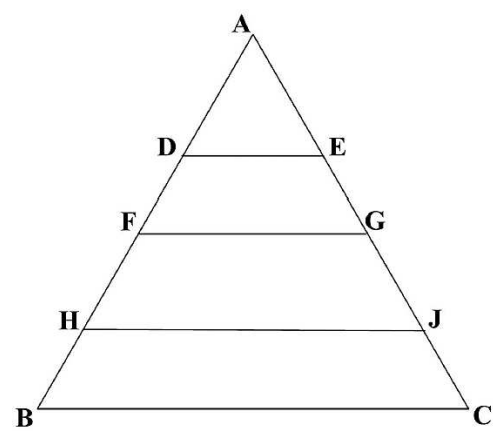

Fig. 1: Cosmic Triangles.

Let $\mathrm{u}, \mathrm{x}, \mathrm{y}$ and $\mathrm{z}$ denote the sum of the interior angles of cosmic triangles ADE, AFG, AHJ and ABC respectively. In another words,

$$
\begin{aligned}
& A D E=u---------------[\mathrm{I}] \\
& A F G=x---------------[\mathrm{II}] \\
& A H J=y---------------[\mathrm{III}] \\
& A B C=z--------------[\mathrm{IV}]
\end{aligned}
$$


Let us assume that

$$
\begin{gathered}
x+y=2 v+s \\
y+z=2 v+t \\
m+z=2 v+u
\end{gathered}
$$

where $2 v, s, t$ and $m$ are real and positive integers.

(2) - (3) gives,

$$
y+u=m+t
$$

Squaring (1),

$$
x^{2}+y^{2}+2 x y=4 v^{2}+s^{2}+4 v s
$$

Squaring (4),

$$
m^{2}+t^{2}+2 m t=y^{2}+u^{2}+2 y u
$$

Adding (5) and ( 6 ),

$$
\begin{aligned}
& x(x+y)+x y+(m+2 v)(m-2 v)+(t+s)(t-s)+2 m t-u(y+u)-y u-4 v s=0 \\
& \text { i.e, } x(x+y)+y(x-u)+2 m t-u(y+u)-4 v s+(t+s)(t-s)=0
\end{aligned}
$$

Applying (3) in the first factor, and (6) in the third factor we have,

$$
\begin{aligned}
& x(2 v+s)+y(x-u)+2 m t-m u-u t-4 v s+(t+s)(t-s)=0 \\
& \text { i.e, } 2 v(x-s)+s(x-2 v)+y(x-u)+m(t-u)+t(m-u)+(t+s)(t-s)=0
\end{aligned}
$$

From (3) we have, $x-s=2 v-y$ and $x-2 v=s-y$. Assuming these values in the above relation

$$
\begin{aligned}
& 2 v(2 v-y)+s(s-y)+y(x-u)+m(t-u)+t(m-u)+(t+s)(t-s)=0 \\
& \text { i.e, } \quad y(x-u-s-2 v)+4 v^{2}+s^{2}+m(t-u)+t(m-u)+(t+s)(t-s)=0
\end{aligned}
$$

Assuming ( 3 ) in the first factor,

$$
-y(y+u)+4 v^{2}+s^{2}+m(t-u)+t(m-u)+(t+s)(t-s)=0
$$

Rearranging, $\left.-y(y+u)+4 v^{2}+m(t-u)+t(m-u+t-s)+s(s+t-s)\right)=0$.

Putting (4) in the first and third factors, $-y(m+t)+4 v^{2}+m(t-u)+t(y-u+u-s)+s(s+t-s)=0$ i.e $-y m+4 v^{2}+$ $m(t-u)=0$, i.e $m(t-u-y)+4 v^{2}=0$. Applying (4) in the first factor, $m(t-m-t)+4 v^{2}=0$, i.e $+4 v^{2}=m^{2}$,

$$
m=2 v
$$

Assuming (7) in (3) we obtain that

$$
z=u
$$

Comparing [I], [IV] and (8) we get that the sum of the interior angles of triangles ADE and ABC are equal. i. e Triangles $\mathrm{ADE}$ and $\mathrm{ABC}$ are similar (10) This shows that The Geometry of Our Universe is flat . [ 1-8 ]

\section{Case 2}


Given: Cosmic Triangle ABC.

Construction: Make $A D=A \mathrm{E} ; A F=A G$ and $A H=A J$. Join $\mathrm{D}$ and $\mathrm{E} ; \mathrm{F}$ and $\mathrm{G}$ and $\mathrm{H}$ and $\mathrm{J}$. et assume that $\mathrm{e}, \mathrm{w}, \mathrm{s}$ and $\mathrm{g}$ denote the sum of the interior angles of cosmic triangles $\mathrm{ADE}, \mathrm{AFG}, \mathrm{AHJ}$ and $\mathrm{ABC}$ respectively i.e. to put in other words,

$$
\begin{aligned}
& A D E=e------------[\mathrm{I}] \\
& A F G=w------------[\mathrm{II}] \\
& A H J=s------------[\mathrm{III}] \\
& A B C=g------------[\mathrm{IV}]
\end{aligned}
$$

\section{Results}

Let

$$
\begin{aligned}
& e+w=k+n \\
& e+g=k+o
\end{aligned}
$$

and

$$
s+g=k+r
$$

wherek, $n$,oandrarepositiveandreal.

(9)-(11) gives

$$
e+w+r=n+s+g
$$

Squaring (12),

$$
\begin{gathered}
e^{2}+w^{2}+r^{2}+2 e w+2 e r+2 w r=n^{2}+s^{2}+g^{2}+2 n s+2 n g+2 s g \\
(e-g)(e+g)+(w-n)(w+n)+(r-s)(r+s)+2 e w+2 e r+2 w r=2 n s+2 n g+2 s g
\end{gathered}
$$

From (9) we get

$$
w-n=k-e
$$

From (11) we have

$$
r-s=g-k
$$

Applying (10), (15) and (16) in the first, second and third factors respectively in (13),

$$
\begin{aligned}
& (e-g)(k+o)+(k-e)(w+n)+(g-k)(r+s)+2 e w+2 e r+2 w r=2 n s+2 n g+2 s g \\
& k[e-g+w+n-r-s]+o(e-g)-e(w+n)+g(r+s)+2 e w+2 r(e+w)=2 n(s+g)+2 s g .
\end{aligned}
$$

Replacing $s+g b y k+r$ and $e+w b y k+n$ [See eqns. (9) and (11)].

$$
k[k+n-k-r+n-r]+o(e-g)-e(w+n)+g(r+s)+2 e w+2 r(k+n)=2 n(k+r)+2 s g .
$$

Simplifying $2 k(n-r)+o(e-g)+e(w-n)+g r+2 r k=2 n k+s g$ i.e.

$$
o(e-g)+e(w-n)+g(r-s)=0
$$


From (9)

$$
w-n=k-e .
$$

From (11)

$$
r-s=g-k
$$

Assuming (18) and (19) in (17), $o(e-g)+e(k-e)+g(g-k)=0$,i.e.

$$
e(k+o)-e^{2}+g^{2}-g(k+o)=0
$$

Eqn. (20) is quadratic in $\mathrm{g}$

$$
g=\frac{k+o \pm\left[(k+o)^{2}+4 e^{2}-4 e(k+o)\right]^{1 / 2}}{2} .
$$

Putting $e+g$ for $k+o$ [See eqn. (10)].

$$
g=\frac{e+g \pm\left[(e+g)^{2}+4 e(e-e-g)\right]^{1 / 2}}{2}
$$

i.e.

$$
g=\frac{e+g \pm(e-g)}{2}
$$

We are totally and purely free from the fetters of the laws of quadratic eqns. To assume positive value in (20), So,

$$
g=\frac{e+g+(e-g)}{2}
$$

and

$$
g=e
$$

From (21) we obtain that the sum of the interior angles of cosmic triangles ABC and ADE are equal.

The summit angle $\mathrm{ABC}$ in triangle $\mathrm{ABC}$ and $\mathrm{ACB}$ of triangle $\mathrm{ABC}$ and the base angles ADE and AED of triangle ADE are equal. So, we get that the cosmic triangles ABC and ADE similar. Similar triangles are not possible in hyperbolic and spherical spaces. So, our finding shows that the sum of the interior angles of the given cosmic triangle $A B C$ is equal to $180^{\circ}$ This clearly indicates that the geometry of our universe is Euclidean. [ 1 - 8].

\section{Case 3}

Given: Cosmic Triangle ABC.

Construction: Make $A D=A E ; A F=A G$ and $A H=A J$. Join $\mathrm{D}$ and $\mathrm{E} ; \mathrm{F}$ and $\mathrm{G}$ and $\mathrm{H}$ and $\mathrm{J}$ Let $\mathrm{u}, \mathrm{x}, \mathrm{y}$ and $\mathrm{z}$ denote the sum of the interior angles of Cosmic triangles ADE, AFG, AHJ and ABC respectively. In another words,

$$
\begin{aligned}
& A D E=u----------[\mathrm{I}] \\
& A F G=x---------[\mathrm{II}] \\
& A H J=y----------[\mathrm{III}] \\
& A B C=z----------[\mathrm{IV}]
\end{aligned}
$$




$$
\begin{aligned}
x+y & =v+a \\
y+z & =v+b \\
z+m & =2 v+c \\
m+x & =2 v+d
\end{aligned}
$$

where $v, 2 v, a, b, c, d$ and $m$ are real positive and positive. (22) - (25) gives,

$$
m+a=y+v+d
$$

(23) - (24) gives ,

$$
m+b=y+v+c
$$

Squaring (26),

$$
m^{2}+a^{2}+2 m a=y^{2}+v^{2}+d^{2}+2 y v+2 y d+2 v d
$$

Squaring (27),

$$
m^{2}+b^{2}+2 m b=y^{2}+v^{2}+c^{2}+2 y v+2 y c+2 v c
$$

$(22)+(24)=(23)+(25)$ implies

$$
a+c=b+d
$$

Squaring (25),

$$
a^{2}+c^{2}+2 c a=b^{2}+d^{2}+2 b d
$$

(28) - (29) gives,

$$
\begin{aligned}
& a^{2}-b^{2}+2 m a-2 m b=d^{2}-c^{2}+2 v(d-c)+2 y(d-c) \\
& a^{2}-b^{2}+2 m a-2 m b=d^{2}-c^{2}+2(d-c)[y+v]
\end{aligned}
$$

Putting (27) in RHS, $a^{2}-b^{2}+2 m a-2 m b=d^{2}-c^{2}+2(d-c)[m+b-c]$, i.e. $a^{2}-d^{2}+m[2 a-2 b-2 d+2 c]-b[b+2 c-$ $2 d]+c[2 d+c-2 c]$. Applying (30) in the second factor of LHS, $(a+d)(a-d)-b[b+2 c-2 d]+c[2 d-c]$. From (30), $a-d=b-c$ and $b-a=c-d$, Putting these in the above equation, $b[2 a-3 b+a+d]+c[2 d-c-a-d]$. Applying (30) in the second factor, $b[2 a-3 b+a+d]-b c=0$, i.e. $b[3 a-3 b+d-c]=0$. From (30) we get that $d-c=a-b$. Applying this $b[4 a-4 b]=0$, i.e.

$$
a=b
$$

Analyzing (22), (23) and (32) we have

$$
z=x
$$

From (33) we obtain that the sum of the interior anglers of cosmic triangles ABC and ADE are equal. By construction these two cosmic triangles are isosceles. So, this gives us that these two cosmic triangles are similar. Similar triangles are not possible in spherical/elliptic and hyperbolic spaces. A brief analysis establishes that the geometry of our universe is flat. This is a mathematical proof for previous experimental and measurement results. [ 1-8]

\section{Case 4}

The density parameter $\Omega$, the curvature parameter $\mathrm{k}$ and the Hubble parameter $\mathrm{H}$ are related as [7].

$$
(1-\Omega)=-k c^{2} / H^{2} R^{2}
$$


If omega less than $1, \mathrm{k}$ is -1 , If omega is equal to $1, \mathrm{k}$ is zero, If omega greater than $1, \mathrm{k}$ is +1 If $\mathrm{k}$ is -1 , the geometry of the universe is open, if it is greater than one, the shape of the universe is closed and the universe obeys Euclidean geometry if $\mathrm{k}$ is equal to zero. Squaring (34),

$$
1+\Omega^{2}-2 \Omega-k^{2} c^{4} / H^{4} R^{4}=0 .
$$

Eqn. (35) is quadratic in $\Omega . \Omega$ the sum of the roots is given by,

$$
\alpha+\beta=-b / a
$$

The product of the roots is given by,

$$
\alpha \beta=c / a=1-k^{2} c^{4} / H^{4} R^{4} .
$$

Squaring (36),

$$
\alpha^{2}+\beta^{2}+2 \alpha \beta=4
$$

Putting (37) in (38), $\alpha^{2}+\beta^{2}+2-2 k^{2} c^{4} / H^{4} R^{4}=4$

i.e

$$
\alpha^{2}+\beta^{2}-2 k^{2} c^{4} / H^{4} R^{4}=2
$$

\section{Analysis of Case (4)}

We have already seen that if $\Omega$ is less than 1 , $\mathrm{k}$ is less than- 1 , if $\Omega$ is equal to 1 , k is zero, and if $\Omega$ is greater than 1 , k is +1 . Since equation (39) is a cosmological one, the roots must be distinct and positive. Assuming $\Omega$ is equal to one, the curvature parameter is zero, the roots $\alpha$ and $\beta$ are distinct and the value is +1 . This satisfies equation (36). And when we assume $\mathrm{k}=0$, in the same equation, we get that the equations satisfies (40). Also, by assuming the roots are distinct in (36), and $\Omega$ is equal to 1 , we get that the equation again satisfies (41). By assuming $\Omega$ is greater than 1 , and the roots are distinct, we get a contradiction in eqn.(36) and (42). By assuming that the roots are distinct and $\Omega$. Is less than 1 . Again we obtain a contradiction (43).

\section{Conclusion of Case 4}

A brief analysis of equation (42) reveals that our universe cannot be open (44). And a brief analysis of equation (43) reveals that our universe cannot be closed (45). So, the mere comparison of eqns. (35) and (40), (36), (40), (43) and (44) (45) establish once and for all that our universe is FLAT [1-8].

\section{Discussion}

In the above four cases, we have seen that the geometry of our universe is flat.[ 1 - 8 ] This is the first mathematical formulation for the geometry of our universe. Further probes may unlock many facts.

\section{Competing interests}

The authors declare that they have no competing interests.

\section{Authors' contributions}

All authors have contributed to all parts of the article. All authors read and approved the final manuscript. 


\section{References}

[1] https://arxiv.org/pdf/astro-ph/9807311.pdf

[2] https://arxiv.org/abs/0802.2236

[3] https://arxiv.org/abs/astro-ph/0412407

[4] https://arxiv.org/abs/astro-ph/9708225

[5] https://arxiv.org/abs/astro-ph/0210279

[6] https://arxiv.org/abs/gr-qc/0105048

[7] https://arxiv.org/abs/astro-ph/0305071

[8] https://arxiv.org/abs/1309.5815 